\title{
SURVEY OF SCALE INSECTS, MEALY BUGS AND ASSOCIATED NATURAL ENEMIES ON MULBERRY TREES IN THE NILE DELTA
}

\author{
HENDAWY, A. S., I. A. I. SAAD and REHAB H. TAHA \\ Plant Protection Research Institute, ARC, Dokki, Giza, Egypt
}

(Manuscript received 14 July 2013)

\begin{abstract}
Sampling of two mulberry tree species namely Morus alba L. and M. nigra L. was conducted from January 2011 to December 2012 to determine the arthropod pests attacking the trees, and their associated natural enemies at Delta region. Two arthropod classes were recorded during the present study (Insecta and Arachnida). Scale insects (Asterolecanium pustulans (Ckll.), Ceroplastes rusci L., Coccus hesperidum L., Saissetia oleae (Bern.), Pseudaulacaspis pentagona (Targ.-Tozz.), Hemiberlesia lataniae Signoret) and mealy bugs (Icerya aegyptiaca (Dough), Icerya purchasi Maskell, Icerya seychellarum (Westw.), Ferrisia virgata (Ckll.), Planococcus citri (Risso), Maconellicoccus hirsutus (Green)) were surveyed as major insect pests. The minor ones were accounted for 24 species (including insects and mites). The parasitoids associated with scale insects and mealy bugs were arranged in five families, Aphytis spp., Encarsia citrina (Crawford), Anagyrus kamali Moursi, Metaphycus sp., Allotropa mecrida (Walker), Scutellista caerulea (Fonscolombe) the most occurring. As for predators, 31 insect species and 9 spiders were found associated with the two pest categories. The predatory mites were identified as two species, which regulate the spider mites. Population fluctuations of mulberry mealy bug, Maconellicoccus hirsutus (Green) were found related to the population fluctuation of Nephus bipunctatus.
\end{abstract}

\section{INTRODUCTION}

Mulberry, Morus alba L. (Moraceae) is a widespread and important fruit tree. It is also used for silkworm feed, and a source of woods in many parts of the world including Egypt. In temperate and sub-tropical climates, mulberries are deciduous, under tropical upland conditions they are in leaf throughout the year (FAO, 2002, and Govindaiah, et al.,2005).

Sericulture is the most important commercial use for white mulberry. Qualitative and quantitative leaves can only ensures quality cocoon and quality silk (Govindaiah, et al.,2005). Morus alba is attacked by many pests (Sanchez, 2000 and Rama and Kumar 2007). The mulberry M. alba and M. nigra are cultivated in Egypt around the roads, houses and farms, many local trees scattered all over the Egyptian country 
side. The mulberry trees have not any protection, pruning and fertilizer treatments. Many mites, insect pests cause a problem on shoots and leaves. Incidence of these pests in around the Egyptian area is unknown.

As the authors aware, no studies were conducted on insect pests of mulberry trees in Egypt. However, Hosny et al.(1976), in a text book, mentioned that mulberry trees as a wood tree are attacked by some insect pests, the Egyptian mealy bug, Icerya aegyptiaca (Douglas), the pink hibiscus mealy bug, Maconellicoccus hirsutus (Green), the fig wax scale Ceroplastes rusci (L.), the pit-making scale, Asterolecanium pustulans (Ckll.), the Egyptian cotton leafworm, Spodoptera littoralis (Boisd.), the large brown longhorn beetle Macrotoma palmata (F.) and the longhorned beetle, Rhaesus serricollis (Motschulsky). Attia, (2012) recorded striped mealy bug, Ferrisia virgata (Cockerell) on Morus alba in Cairo and Giza Governorates parasitized by Blepyrus insularis.

Mulberry mealy bug, Maconellicoccus hirsutus (Hemiptera: Pseudococcidae) infestation of white and black mulberry causes malformation of terminal buds and appearance of small curly leaves on the shoots and top apical. M. hirsutus is a serious polyphagous pest feeding voraciously on mulberry leaves (Manjunath et al., 2006, Mala et al., 2007). The pest has been found on 215 genera of plants. Its wide host range favors rapid and complicated effective control. A heavy black sooty mold sometimes develops on infested leaves and stems as a result of the mealy bug heavy secretion was described by Alleyne, (2004). M. hirsutus was considered an economic pest in many of the tropical and semi tropical regions of the world (CABI/EPPO, 2004). Many Egyptian studies have addressed the natural enemies of scale insects and mealy bugs (Hendawy 1999, Abd-Rabou and Hendawy 2000, Mousa et al 2001, Hendawy et al 2002, Abd-Rabou and Hendawy 2005). The current study aimed to survey the local pests attacking the mulberry trees and their natural enemies in Delta Region of Egypt.

\section{MATERIAL AND METHODS}

Surveys were carried out at Delta region (Kafr El-Sheikh and Gharbia governorats) from January 2011 to December 2012 on mulberry trees, Morus alba L. and $M$. nigra L. to determine the arthropods attacking these trees and their natural enemies. The trees aged $3-10$ years old and scattered in plantations of annual crops (rice, maize, cotton, soybean, bean, clover, sugar beet, flax and wheat), around citrus and table grape orchards, water canals and roads. The considered trees were not treated with any of insecticides throughout the experimental period because most of trees are close to irrigation canals and rural roads. 


\section{Sampling of arthropods:}

\section{Parasitoids:}

Monthly samples (January $1^{\text {st }}, 2011$ up to December $30^{\text {th }}, 2012$ ) were taken from mulberry trees at Sakha and Kotoor areas. At each location, five mulberry trees were assigned, throughout the experimental period, for sampling. When sampling, 20 leaves and 4 branches (shoots) were picked up from a tree. Each tree was sampled from the four cardinal directions, east, west, north and south, as five leaves and one branch per direction. Thus, at each sampling date, 100 leaves and 20 branches were obtained from a location. The collected leaves and shoots were introduced into paper bags, and transferred to the insect laboratory, Sakha Agricultural Research Station for examinations.

Leaves having parasitoid insects only were separated from other insect infestations. Then, they were introduced into wooden boxes (50 $20 \times 20 \mathrm{~cm}$.) to collect the emerging parasitoids. A glass tube was inserted into a round hole of the box. An electric lamp $(60 \mathrm{w}$.) was placed in front of the box to attract emerging parasitoids, and enter the glass tube. Emerging parasitoids were identified and monitored and collected continuously by substituting the tubes having the parasitoids by new ones. Also, mulberry leaves were replaced by new ones having alive insects every two weeks.

\section{Predators :}

The insect pests, insect predators and spiders associated with mulberry insects were surveyed. Monthly samples were taken from the same five trees assigned for sampling insect pests, and at the same day. When sampling, the arthropods of four branches per tree were excluded by introducing the branch into a white cloth bag (50 cm long). Then, the branch was strongly beaten to drop the occurring arthropods inside the cloth bag. This procedure was conducted for the four branches of a tree. Thus, the arthropods were collected from 20 branches/ location/ sample. The cloth bag was thoroughly closed, transferred to the laboratory at Sakha Agricultural Research Station and kept in the refrigerator to anesthetize the confined arthropods. Fifteen minutes later, the bag was taken out from the refrigerator and emptied to examine and count the insect pests and natural enemies. Afterwards, specimens were kept in insect wooden boxes or into glass vials with $70 \%$ ethyl alcohol, and two drops of glycerin to keep the arthropod tissues soft, to be easily handled, and identified. 


\section{Examination and Identification:}

Monthly, the 100 leaves and twenty five branches of mulberry of a location were examined, and numbers of arthropods were recorded using a binocular microscope. Specimens of mites, insect pests, predators and emerging parasitoids were separated and initially identified, then sent to the Systematic Research Department and Biological Control Research Department at Plant Protection Research Institute, Giza, Egypt for confirming the identification.

\section{Relationship between Maconellicoccus hirsutus and its predator, Nephus bipunctatus}

During observations in Kafr-El Sheikh governorate, larvae and adults of Nephus bipunctatus (Kugelann) were found feeding voraciously on all stages of the $M$. hirsutus. Seasonal distribution of $M$. hirsutus and adult of $N$. bipunctatus was recorded on white mulberry from May to October, 2011. Randomly 10 shoots $(20 \mathrm{~cm}$. length) were taken from 5 mulberry trees in four direction, Kept in paper and polyethylene bags and transferred to laboratory to count the population of mulberry mealy bug (MMB) and its predator.

\section{RESULTS AND DISCUSSION}

Arthropod pests inhabiting mulberry trees were surveyed on mulberry trees at Kafr El-Sheikh and Gharbia Governorates (Table 1).

Three mite species belonging to two families, Tenuipalpidae (one species) and two species were related to Tetranychidae. These species were detected from May June or from May - September, according to the mite species. The surveyed arthropods were categorized regardless of the governorates. Thus, the listed insect pests, parasitoids and predators, were presented occurring in both locations. Twenty one insect species, belonging to thirteen families and five orders, were surveyed. Two species are related to order Thysanoptera by two families and two species (AprilMay). Order Hemiptera have two families and three species, Nezara viridula was detected from May to October. Homoptera is represented by four species and three families. Lepidoptera and Coleoptera are represented by six species for each. Lepidoptera have three families, Noctuidae (4 species), Phycitidae and Lyonetiidae one species for each. Coleoptera is represented by three families, Scarabaeidae (2 species), Cerambycidae (3 species) and Scolytidae (one species). 
Table 1. Occurrence of minor arthropod pests attacking mulberry trees at Delta region from January 2011 to December 2012

\begin{tabular}{|c|c|c|}
\hline Order/ Family & Species & Period of occurrence \\
\hline $\begin{array}{l}\text { Acarina } \\
\text { Tenuipalpidae }\end{array}$ & Brevipalpus sp. & May- June \\
\hline Tetranychidae & $\begin{array}{l}\text { Panonychus ulmi (Koch) } \\
\text { Tetranychus urticae } \\
\text { Koch }\end{array}$ & $\begin{array}{l}\text { May- June } \\
\text { May-Sept. }\end{array}$ \\
\hline \multicolumn{3}{|l|}{ Insecta } \\
\hline $\begin{array}{l}\text { Thysanoptera } \\
\text { Phlaeothripidae }\end{array}$ & Haplothrips sp. & April- May \\
\hline Thripidae & Thrips tabaci Lindman & April- May \\
\hline \multicolumn{3}{|l|}{ Hemiptera } \\
\hline Miridae & Campylomma sp. & May \\
\hline Pentatomidae & $\begin{array}{l}\text { Agonoscelis pubescens } \\
\text { Thunb. } \\
\text { Nezara viridula (L.) }\end{array}$ & $\begin{array}{l}\text { May } \\
\text { May-October }\end{array}$ \\
\hline \multicolumn{3}{|l|}{ Homoptera } \\
\hline Aleyrodidae & Bemisia tabaci (Genn.) & April- May \\
\hline Aphidae & $\begin{array}{l}\text { Aphis gossypii Glov. } \\
\text { Myzus persicae (Sulz.) }\end{array}$ & $\begin{array}{l}\text { June-August } \\
\text { June-August }\end{array}$ \\
\hline Cicadellidae & Empoasca decipiens Paoli & June-August \\
\hline \multicolumn{3}{|l|}{ Lepidoptera } \\
\hline Noctuidae & $\begin{array}{l}\text { Autographa gamma (L.) } \\
\text { Trichoplusia ni (Hbn.) } \\
\text { Spodoptera exigua (Hbn.) } \\
\text { Spodoptera littoralis (Boisd.) }\end{array}$ & $\begin{array}{l}\text { May } \\
\text { May } \\
\text { April- September } \\
\text { April- October } \\
\end{array}$ \\
\hline Phycitidae & $\begin{array}{l}\text { Cryptoblabes gnidiella } \\
\text { (Mill.) }\end{array}$ & April-May \\
\hline Lyonetiidae & Lyonetia clerkella (L.) & May \\
\hline \multicolumn{3}{|l|}{ Coleoptera } \\
\hline Scarabaeidae & $\begin{array}{l}\text { Pachnoda fasciata (F.) } \\
\text { Tropinota squalida (Scop.) }\end{array}$ & $\begin{array}{l}\text { March } \\
\text { March -April }\end{array}$ \\
\hline Cerambycidae & 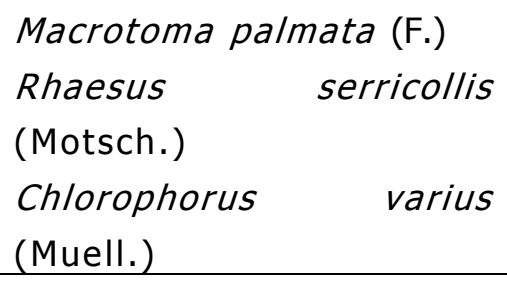 & $\begin{array}{l}\text { July on M. alba } \\
\text { July on M. alba } \\
\text { Jul., Aug. on M. alba \& } \\
\text { M.nigra }\end{array}$ \\
\hline Scolytidae & $\begin{array}{l}\text { Scolytus rugulosus } \\
\text { (Muller) }\end{array}$ & Dead wood \\
\hline
\end{tabular}




\section{Survey of scale insects and mealy bugs attacking mulberry trees:}

Six species of scale insects were surveyed from mulberry trees. The scale insects are related to Asterolecaniidae, Asterolecanium pustulans, three Coccidae, Ceroplastes rusci, Coccus hesperidium and Saissetia oleae, and two Diaspididae, Pseudaulacaspis pentagona and Hemiberlesia lataniae. Three species of Pseudococcidae and three Margarodids, were surveyed at Kafr El-Sheikh and Gharbia Governorates (Table 2 ).

Scale insects and mealy bugs were previously surveyed from the Egyptian mulberry trees, Icerya aegyptiaca, Maconellicoccus hirsutus, Ceroplastes rusci, Asterolecanium pustulans (Hosny et al, 1976), Ferrisia virgata (Attia 2012).

Table 2. Scale insects and mealy bugs attacking mulberry trees at Delta region from January 2011 to December 2012

\begin{tabular}{|c|c|c|}
\hline Family & Species & $\begin{array}{l}\text { Period of } \\
\text { occurrence }\end{array}$ \\
\hline Asterolecaniidae & Asterolecanium pustulans (Ckll.) & January-December \\
\hline Coccidae & $\begin{array}{l}\text { Ceroplastes rusci L. } \\
\text { Coccus hesperidum L. } \\
\text { Saissetia oleae (Bern.) }\end{array}$ & $\begin{array}{l}\text { June-August } \\
\text { June-August } \\
\text { June-August }\end{array}$ \\
\hline Diaspididae & $\begin{array}{l}\text { Pseudaulacaspis pentagona } \\
\text { (Targ.-Tozz.) } \\
\text { Hemiberlesia lataniae Signoret }\end{array}$ & $\begin{array}{l}\text { January-December } \\
\text { May - October }\end{array}$ \\
\hline Margarodidae & $\begin{array}{l}\text { Icerya aegyptiaca (Dough) } \\
\text { Icerya purchasi Maskell } \\
\text { Icerya seychellarum (Westw.) }\end{array}$ & $\begin{array}{l}\text { May - October } \\
\text { May - October } \\
\text { May - October }\end{array}$ \\
\hline Pseudococcidae & $\begin{array}{l}\text { Ferrisia virgata (Ckll.) } \\
\text { Planococcus citri (Risso) } \\
\text { Maconellicoccus hirsutus (Green) }\end{array}$ & $\begin{array}{l}\text { May - October } \\
\text { May - October } \\
\text { May - October }\end{array}$ \\
\hline
\end{tabular}

\section{Survey of parasitoids:}

Seventeen species of super family Chalcidoidea belonging to five families and ten genera were collected during the period of study (January, 2011 till December, 2012) associated with mulberry armored, soft scales and mealy bugs. The obtained results in Table (3) show the parasitoid species and their hosts. 
Table 3. Hymenopterous parasitoids attacking mulberry scale insects and mealy bugs in Delta region from January 2011 to December 2012

\begin{tabular}{|c|l|l|}
\hline Family & \multicolumn{1}{|c|}{ Parasitoid } & \multicolumn{1}{|c|}{ Host species } \\
\hline \multirow{5}{*}{ Aphelinidae } & Aphytis spp. & $\begin{array}{l}\text { Pseudaulacaspis } \\
\text { pentagona } \\
\text { Hemiberlesia lataniae }\end{array}$ \\
\cline { 2 - 3 } & Encarsia citrina (Crawford) & $\begin{array}{l}\text { Pseudaulacaspis } \\
\text { pentagona } \\
\text { Hemiberlesia lataniae }\end{array}$ \\
\cline { 2 - 3 } & Coccophagus scutellaris (Dalman) & Coccus hesperidum \\
\cline { 2 - 3 } & Marietta leopardina Motschulsky* & Aphytis spp. \\
\hline \multirow{5}{*}{ Encyrtidae } & Anagyrus kamali Moursi & Maconellicoccus hirsutus \\
\cline { 2 - 3 } & Anagyrus seudococci (Girault) & Maconellicoccus hirsutus \\
\cline { 2 - 3 } & Blepyrus insularis (Cameron) & Ferrisia virgata \\
\cline { 2 - 3 } & Clausenia josefi (Rosen) & Maconellicoccus hirsutus \\
\cline { 2 - 3 } & $\begin{array}{l}\text { Coccidoxenoides } \\
\text { (Timberlake) }\end{array}$ & Planococcus citri \\
\cline { 2 - 3 } & Gyranusoidea sp. & Maconellicoccus hirsutus \\
\cline { 2 - 3 } & Leptomastidea abnormis (Girault) & Planococcus citri \\
\cline { 2 - 3 } & Leptomastix dactylopii Howard & Maconellicoccus hirsutus \\
\cline { 2 - 3 } & Metaphycus sp. & $\begin{array}{l}\text { Ceroplastes floridensis } \\
\text { Saissetia oleae }\end{array}$ \\
\cline { 2 - 3 } & Microterys sp. & Coccus hesperidum \\
\hline Signiphoridae & Chartocerus sp. * & Maconellicoccus hirsutus \\
\hline Platygastridae & Allotropa mecrida (Walker) & $\begin{array}{l}\text { Ceroplastes rusci } \\
\text { Saissetia oleae }\end{array}$ \\
\hline \multirow{2}{*}{ Pteromalidae } & Scutellista caerulea (Fonscolombe) &
\end{tabular}

* hyperparasitoid

\section{Survey of predaceous insects:}

Thirty one insect predators, belonging to six orders and 11 families were surveyed from mulberry trees. Most of surveyed species are belonging to coleopteran, mainly Coccinellidae. The important predators are Chilocorus bipustulatus L., Exochomus flavipes Thunb., Hyperaspis sp., Nephus includens (Kirsch),Nephus bipunctatus (Kugelann), Pharoscymnus sp., Rhizobius litura F., Rodolia cardinalis (Mulsant), Pullus syriacus Mulsant, Cybocephalus sp. as they prey upon scale insects and mealy bugs. Family Coccinellidae was represented by 16 predatory species surveyed in the current study (Table 4). This family was often detected by several Egyptian authors when surveyed the scale insects and mealy bugs predators in fruit trees, e.g. Priesner. and Hosny,1940 and Hendawy 1999. In addition, two neuropterous species, Conwentzia psociformis (Curtis), Chrysoperla carnea Steph. prey upon the sam insect pests. 
Table 4. Predatory insects inhabiting mulberry trees at Delta region from January 2011 to December 2012

\begin{tabular}{|c|c|c|}
\hline Order/ Family & Species & Prey \\
\hline $\begin{array}{l}\text { Thysanoptera } \\
\text { Aeolothripidae }\end{array}$ & Aeolothrips sp. & Thrips, Mites \\
\hline $\begin{array}{l}\text { Hemiptera } \\
\text { Anthocoridae }\end{array}$ & $\begin{array}{l}\text { Orius laevigatus (Fieber) } \\
\text { Orius albidipennis (Reuter) }\end{array}$ & $\begin{array}{l}\text { Aphids, scale insects, } \\
\text { mealy bugs, thrips, } \\
\text { mites }\end{array}$ \\
\hline Reduviidae & Coranus sp. (F.) & Moth \\
\hline $\begin{array}{l}\text { Coleoptera } \\
\text { Coccinellidae }\end{array}$ & $\begin{array}{l}\text { Chilocorus bipustulatus L. } \\
\text { Coccinella undecimpunctata } \\
\text { L. } \\
\text { Cydonia vicina Isis Cr. } \\
\text { Cydonia vicina nilotica Muls. } \\
\text { Cydonia vicina subsignata Pic } \\
\text { Exochomus flavipes Thunb. } \\
\text { Hippodamia convergens G.-M. } \\
\text { Hyperaspis sp. } \\
\text { Nephus includens (Kirsch) } \\
\text { Nephus bipunctatus } \\
\text { (Kugelann) } \\
\text { Pharoscymnus sp. } \\
\text { Stethorus gilvifrons Mulsant } \\
\text { Rhizobius litura F. } \\
\text { Rodolia cardinalis (Mulsant) } \\
\text { Scymnus interruptus (Goeze) } \\
\text { Pullus syriacus Mulsant }\end{array}$ & $\begin{array}{l}\text { scale insects } \\
\text { Aphids } \\
\text { Aphids } \\
\text { Aphids } \\
\text { Aphids } \\
\text { Aphids, Mealy bugs } \\
\text { Aphids } \\
\text { Aphids, Mealy bugs } \\
\text { Mealy bugs } \\
\text { Aphids, mealy bugs } \\
\text { Mealy bugs } \\
\text { Mites } \\
\text { Aphids, } \\
\text { Icerya spp. } \\
\text { Aphids } \\
\text { Aphids, Mealy bugs }\end{array}$ \\
\hline Carabidae & $\begin{array}{l}\text { Bembidion sp.* } \\
\text { Tachys sp.* }\end{array}$ & Aphids \\
\hline Cybocephalidae & Cybocephalus sp. & scale insects \\
\hline Staphylinidae & $\begin{array}{l}\text { Paederus alfierii Koch. } \\
\text { Philonthus spp. }\end{array}$ & $\begin{array}{l}\text { Aphids } \\
\text { Aphids }\end{array}$ \\
\hline $\begin{array}{l}\text { Diptera } \\
\text { Syrphidae }\end{array}$ & $\begin{array}{l}\text { Eupeodes corollae (F.) } \\
\text { Paragus compeditus (Wied.) } \\
\text { Sphaerophoria rueppelli } \\
\text { (Wied.) }\end{array}$ & $\begin{array}{l}\text { Aphids } \\
\text { Aphids } \\
\text { Aphids }\end{array}$ \\
\hline $\begin{array}{l}\text { Neuroptera } \\
\text { Coniopterygidae } \\
\text { Chrysopidae }\end{array}$ & $\begin{array}{l}\text { Conwentzia psociformis (Curtis) } \\
\text { Chrysoperla carnea Steph. }\end{array}$ & $\begin{array}{l}\text { Mites, mealy bugs } \\
\text { Aphids, scale insects, } \\
\text { mealy bugs, thrips, } \\
\text { mites }\end{array}$ \\
\hline $\begin{array}{l}\text { Orthoptera } \\
\text { Mantidae }\end{array}$ & Mantis religiosa L. & $\begin{array}{l}\text { aphids, fruit flies, flies, } \\
\text { moths }\end{array}$ \\
\hline
\end{tabular}

* Around tree trunks 


\section{Survey of predaceous mites:}

Two predacious mites, belonging to family Phytoseiidae, and nine species, belonging to eight families of spiders, Araneidae, Dyctinidae, Miturigidae, Lycosidae, Pholcidae Salticidae, Theridiidae and Thomisidae, were surveyed (Table 5 ).

Table 5 . Predacious mites and spiders collected on mulberry trees at delta region from January 2011 to December 2012

\begin{tabular}{|c|c|c|}
\hline Family/Common name & Species & $\begin{array}{l}\text { Period of } \\
\text { occurrence }\end{array}$ \\
\hline $\begin{array}{l}\text { Acarina } \\
\text { Phytoseiidae }\end{array}$ & $\begin{array}{l}\text { Amblyseius sp. } \\
\text { Typhlodromus sp }\end{array}$ & $\begin{array}{l}\text { May- July } \\
\text { May-Sept. }\end{array}$ \\
\hline \multicolumn{3}{|l|}{ Araneae } \\
\hline $\begin{array}{l}\text { Araneidae } \\
\text { weaver }\end{array}$ & Cyrtophora citricola Forskål & July. \\
\hline $\begin{array}{l}\text { Dictynidae } \\
\text { Mesh web weaver }\end{array}$ & Dictyna sp. & Jun., Jul. \\
\hline $\begin{array}{l}\text { Miturigidae } \\
\text { Long-legged sac spider }\end{array}$ & Chiracanthium sp. & May, Jun., July \\
\hline $\begin{array}{l}\text { Lycosidae } \\
\text { Wolf spider }\end{array}$ & Pardosa spp.* & May, June, July \\
\hline $\begin{array}{l}\text { Pholcidae } \\
\text { Daddylonglegs spiders }\end{array}$ & $\begin{array}{l}\text { Pholcus phalangioides } \\
\text { (Fuesllin)** }\end{array}$ & Aug-Oct. \\
\hline $\begin{array}{l}\text { Salticidae } \\
\text { Jumping spider }\end{array}$ & $\begin{array}{l}\text { Plexippus paykulli Audouin ** } \\
\text { Thyene imperialis (Rossi) }\end{array}$ & $\begin{array}{l}\text { May-Sept. } \\
\text { May-Sept. }\end{array}$ \\
\hline $\begin{array}{l}\text { Theridiidae } \\
\text { Comb-footed spider }\end{array}$ & Theridion sp. & June, July \\
\hline $\begin{array}{l}\text { Thomisidae } \\
\text { Crab spider }\end{array}$ & Thomisius sp. *** & March \\
\hline
\end{tabular}

* Around tree trunks $\quad * *$ Beside the houses $* * *$ on flowers

Periodical observations were recorded on the populations of mulberry mealy bug, M. hirsutus and its coccinellid predator, N. bipunctatus on mulberry shoots in mulberry trees from May to October, 2011. The mealy bug population raised during the period from June to September with the peak incidence of the pest observed 
during second fortnight of June. During May to October, activity of other natural enemies could not be seen in the mulberry trees with the coccinellid predator, $N$. bipunctatus being the dominant predator feeding on the of mealy bug ovisacs. During September and October, presence of coccinellids like Nephus includens Kirsch and Pullus syriacus Marseul. could be noticed in the mulberry trees and the population of N. bipunctatus was declining (Table 6).

Table 6. Population fluctuations of the mulberry mealy bug, Maconellicoccus hirsutus and adult of coccinellid predator Nephus bipunctatus in mulberry tree at Kafr El- Sheikh Governorate (from May to October, 2011)

\begin{tabular}{|l|c|c|}
\hline \multirow{2}{*}{ Date of collection } & \multicolumn{2}{|c|}{ Population / Shoot $20 \mathrm{~cm}$} \\
\cline { 2 - 3 } & M. hirsutus & N. bipunctatus \\
\hline $25^{\text {th }}$ May & $2.46 \pm 56.86$ & $2.00 \pm 1.00$ \\
\hline $20^{\text {th }}$ June & $50.60 \pm 23.79$ & $1.30 \pm 0.57$ \\
\hline $25^{\text {th }}$ July & $34.33 \pm 5.03$ & $2.00 \pm 0.75$ \\
\hline $20^{\text {th }}$ August & $24.22 \pm 2.44$ & $1.5 \pm 0.55$ \\
\hline $25^{\text {th }}$ September & $16.33 \pm 4.50$ & $0.66 \pm 0.57$ \\
\hline $20^{\text {th }}$ October & $8.00 \pm 4.36$ & $0.33 \pm 0.58$ \\
\hline
\end{tabular}

The values are mean \pm SD of 10 shoots(top apical)

\section{REFERENCES}

1. Abd-Rabou, S. and A.S. Hendawy. 2000. Parasitoids attacking the parlatoria date scale, Parlatoria blanchardi (Targioni-Tozzetti) (Homoptera : Diaspididae in Egypt. J. Agric. Mansoura Univ.,25(12):8217-8222.

2. Abd-Rabou, S. and A.S. Hendawy. 2005 . Updating nomination of parasitoids of pink hibiscus mealy bug, Maconellicoccus hirsutus (Green), (Homoptera : Pseudococcidae) in Egypt. Egypt. J. Agric. Res. 83 (3),1135-1139.

3. Alleyne J.C. 2004. Controlling a dangerous pest-pink hibiscus mealy bug. Newsletter of the University of Florida - IFAS Extension -Pinellas County Extension, 38(4):2-3.

4. Attia, A. R. 2012. Hymenptrous parasitoids as a bioagents for controlling mealy bugs (Hemiptera: Pseudococcidae) in Egypt. Egypt. Acad. J. Biolog. Sci., 5(3): $183-192$

5. CABI/EPPO. 2004. Maconellicoccus hirsutus, distribution map of plant pests, pp. Map 100. CAB International, Wallingford (GB). 
6. FAO. 2002. Mulberry for Animal production, FAO Animal Production and Health Paper 147 Rome. p. 331.

7. Govindaiah, V. P. Gupta, D. D. Sharma, S. Rajadurai and V. Nishita Naik 2005. A Text Book on Mulberry Crop Protection. Sampath, J. (ed.), Central Silk Board, Bangalore, India.

8. Hendawy, A.S.A. 1999. Studies of certain natural enemies of scale insects attacking guava trees at Kafr El-Sheikh Governorate. Ph. D. Thesis, Fac. Agric., Tanta Univ., 160 pp.

9. Hendawy, A. S, M. F. El-Mitwally and S.A. Abd Elsameh. 2002. Coccophagus scutellaris (Dalman) (Hymenoptera: Aphelinidae), a new record parasitoid attacking sugar-cane soft scale insect, Pulvinaria Tenuivalvata (Newstead) in Egypt. Egypt. J. Agric. Res. 80 (1): 222-224.

10. Hosny, M. M. , M. A. Assem and E. A. Nasr. 1976. Insect and animal agricultural pests.( $2^{\text {ed }}$ edition, Dar El-Maarif, Egypt, p. 1076). (In Arabic).

11. Mala, V.R., K.S. Prasad, D. Manjunath, S. B. Dandin. 2007. Evaluation of germplasm genotypes of mulberry for reaction of sucking pests. Indian J. Sericulture. 46(1): 38-42.

12. Manjunath D., K. S. Prasad, D.K.S. Gowda. 2006. Ecological approach for the management of the mealy bug, Maconellicoccus hirsutus causing tukra in mulberry. Plant Archives. 6(2): 767-768.

13. Mousa, S.F, A.H. El-Heneidy, A.S. Hendawy, D. Adly, D. Gonzalez and S.V. Trjaptsyn. 2001. Pink hibiscus mealy bug , Maconellicoccus hirsutus (Green), Parasitoids in Egypt. 1- Preliminary record. Egypt.J. Biol. Pest Control Vol.11(2) ,195-196.

14. Priesner, A. and M. Hosny.1940. Notes on parasites and predators of Coccidae and Aleyrodidae in Egypt. Bull. Soc. Ent. Egypt, 24: 58-70.

15. Rama K. and K. V. Kumar. 2007. Impact of climatic conditions on the infestation of major pests of mulberry (Morus spp.) in Doon Valley.J. Entomol. Res. Society. 9(2): 17-30.

16. Sanchez M. D. 2000. Mulberry for Animal Production. FAO Animal Production and Health Paper 147. Rome, 331 pp. 


\section{حصر مفصليات الأرجل علي أثجار التوت في منطقة الالتا مع الإشارة بوجه خاص إلى الحثرات القشرية والبق الدقيقي والأعداء الحيوية المرتبطة بها أحمد سمير هنداوي ، إبراهيم عبد العظيم إبراهيم سعد ، رحاب حسني طــه معهُ بحوث وقابة النباتات، مركز البحوث الزراعية ، دقى - مصر}

في أول دراسة مصرية لحصر مفصليات الأرجل علي أنثجار التوت (الأبيض و الأسود)

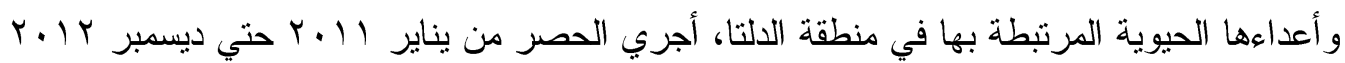

خلا هذه الدراسة سجل صنفين من مفصليات الأرجل (الحشرات و العنكبوتيات). كانت الحشرات القشرية و البق الدقيقي (r ا نوعا) هي الآفات الرئيسية لأشجار التوت أما الآنات الثانوية فتمثل ؟r نوعا من الحشرات و الأكاروسات.

تم حصر IV نوعا من الطفيليات المرتبطة بالحشرات القترية والبق الدقيقي و كانت تتبع خمس

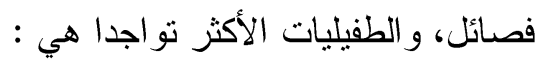

Aphytis spp., Encarsia citrina, Anagyrus kamali, Metaphycus spp., Allotropa mecrida, Scutellista caerulea .

كذا تم حصر اس نوعاً من الحشرات المفترسة، و 9 أنو اع من العناكب الحقبقية المرتبطة بهذه

$$
\text { الآفات، كما نم التعرف على نوعين من الأكاروسات المفترسة. }
$$

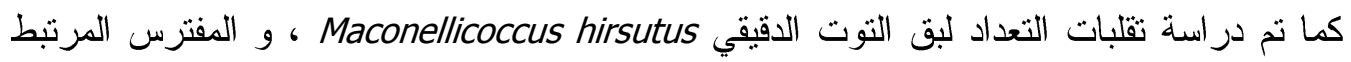

